http://www.mathematik.uni-karlsruhe.de/ ${ }_{\text {semlv }}$

Seminar LV, No. 8, 10 pp., 27.01.2001

\title{
Differential inequalities
}

\author{
Gerd Herzog, Roland Lemmert
}

1. Introduction. Consider a function $f:[a, b] \times \mathbb{R} \rightarrow \mathbb{R}$ and differentiable functions $u, v:[a, b] \rightarrow \mathbb{R}$. It is well known [17], p.65 that

$$
u^{\prime}(t)-f(t, u(t))<v^{\prime}(t)-f(t, v(t)) \quad(t \in[a, b]), \quad u(a)<v(a)
$$

imply $u(t)<v(t)(t \in[a, b])$. What about $\leq$ instead of $<$ everywhere? For example $u(t)=t^{2}, v(t)=0$ satisfy

$$
0=u^{\prime}(t)-2 \sqrt{|u(t)|}=v^{\prime}(t)-2 \sqrt{|v(t)|}(t \in[0,1]), \quad u(0)=v(0)=0
$$

but here $u(t)>v(t)(t \in(0,1])$. On the other hand replacing $<$ by $\leq$ is allowed in case $f$ is for example Lipschitz continuous in its second variable [17], p.69.

May these results be generalized to several variables or even infinite dimensions? The answer is known [14], but is maybe not common knowledge outside the ODE community. We want to present here, in the finite dimensional setting, some simple facts on differential inequalites and its applications, for example to fixed point theory.

2. Norm and order. To get results on inequalities for vector valued functions we have to say what $x \leq y$ means in case that $x, y \in \mathbb{R}^{n}$. Consider a closed and convex subset $K$ of $\mathbb{R}^{n}$ with the following properties:

$$
\lambda K \subseteq K(\lambda \geq 0), \quad K \cap(-K)=\{0\} .
$$

Such a set is called a cone and typical examples are $\left(x=\left(x_{1}, \ldots, x_{n}\right)\right)$

$$
K_{\text {nat }}:=\left\{x \in \mathbb{R}^{n}: x_{1} \geq 0, \ldots, x_{n} \geq 0\right\}
$$

the natural cone,

$$
K_{i c e}:=\left\{x \in \mathbb{R}^{n}: x_{n} \geq \sqrt{x_{1}^{2}+\cdots+x_{n-1}^{2}}\right\}
$$


the $n$-dimensional ice-cream cone, and

$$
K_{\text {pol }}:=\left\{x \in \mathbb{R}^{n}: x_{1}+x_{2} s+\cdots+x_{n} s^{n-1} \geq 0(s \in \mathbb{R})\right\},
$$

which we will call the polynomial cone.

A cone $K$ is called solid if its interior $K^{\circ}$ is not empty. For example $K_{\text {nat }}$ and $K_{i c e}$ are solid cones and $K_{\text {pol }}$ is solid in case $n$ is odd. In the sequel we will only consider solid cones.

If $K$ is a cone we can define a partial ordering on $\mathbb{R}^{n}$ by

$$
x \leq y \Longleftrightarrow y-x \in K,
$$

and we write $x \ll y$ for $y-x \in K^{\circ}$. In case $x \leq y$ we define the order interval $[x, y]$ by $\left\{z \in \mathbb{R}^{n}: x \leq z \leq y\right\}$, which is the same as $(x+K) \cap(y-K)$.

It is useful to connect an ordering to a norm. We fix $p \in K^{\circ}$. The order interval $[-p, p]$ is a closed, bounded and absolut convex set with nonempty interior and is therefore the unit ball of a norm $\|\cdot\|$. For example $p=(1, \ldots, 1) \in$ $\left(K_{\text {nat }}\right)^{\circ}$ leads to the maximum norm, and for $p=(0, \ldots, 0,1) \in\left(K_{\text {ice }}\right)^{\circ}$ we get $\|x\|=\left|x_{n}\right|+\sqrt{x_{1}^{2}+\cdots+x_{n-1}^{2}}$. We have the following compatibility properties between norm and order:

1. $0 \leq x \leq y \Rightarrow\|x\| \leq\|y\|\left(x, y \in \mathbb{R}^{n}\right)$.

2. $-y \leq x \leq y \Rightarrow\|x\| \leq\|y\|\left(x, y \in \mathbb{R}^{n}\right)$.

3. $-\lambda p \leq x \leq \lambda p \Leftrightarrow\|x\| \leq \lambda\left(x \in \mathbb{R}^{n}, \lambda \geq 0\right)$.

4. $-\|y\| p \leq x \leq\|y\| p \Leftrightarrow\|x\| \leq\|y\|\left(x, y \in \mathbb{R}^{n}\right)$.

5. $[x, y]$ is a bounded and convex set $\left(x, y \in \mathbb{R}^{n}, x \leq y\right)$.

6 . If $\left(x_{n}\right)$ is a sequence in $\mathbb{R}^{n}$ and $x_{1} \leq x_{2} \leq \cdots \leq y$ for some $y \in \mathbb{R}^{n}$, then $\left(x_{n}\right)$ is convergent.

We will not give a proof of these properties here, for the details see [2], $\S 19$. At least for $K_{\text {nat }}$ and $p=(1, \ldots, 1)$ the relations above are quite obvious.

3. Quasimonotonicity. Let $\langle\cdot, \cdot\rangle$ denote the standard inner product on $\mathbb{R}^{n}$. The set

$$
K^{*}:=\left\{\xi \in \mathbb{R}^{n}:\langle x, \xi\rangle \geq 0 \quad(x \in K)\right\}
$$

is a cone since $K$ is solid, and is called the dual cone of $K$. For example $K_{\text {nat }}^{*}=K_{\text {nat }}$ and $K_{i c e}^{*}=K_{\text {ice }}$. In general $K^{*} \neq K$. Now, consider $x \in \mathbb{R}^{n}$. We have $x \geq 0$ if and only if $\langle x, \xi\rangle \geq 0$ for each $\xi \in K^{*}$. If $x \gg 0$ then $\langle x, \xi\rangle>0$ for each $0 \neq \xi \in K^{*}$. On the other hand, if $x$ is in the boundary of $K$ then $\langle x, \xi\rangle=0$ for some $0 \neq \xi \in K^{*}$.

A function $g: \mathbb{R}^{n} \rightarrow \mathbb{R}^{n}$ is called quasimonotone increasing (qmi) [14] if it has the following property: 
If $x, y \in \mathbb{R}^{n}$ and $\xi \in K^{*}$ are such that $x \leq y$ and $\langle x, \xi\rangle=\langle y, \xi\rangle$, then $\langle g(x), \xi\rangle \leq\langle g(y), \xi\rangle$.

This definition calls for some comments. If $g: \mathbb{R}^{n} \rightarrow \mathbb{R}^{n}$ is monotone increasing (that is $x \leq y \Rightarrow g(x) \leq g(y)$ ), then $g$ is qmi. Moreover $x \mapsto \lambda x$ is qmi for each $\lambda \in \mathbb{R}$. Hence $g$ is qmi for example if $g+\lambda$ id is increasing for some $\lambda \in \mathbb{R}$. But this is far from the general case since in the one dimensional case $(K=[0, \infty))$ every function $g: \mathbb{R} \rightarrow \mathbb{R}$ is qmi. The most common case is $\mathbb{R}^{n}$ ordered by $K_{\text {nat }}$. In this case $g=\left(g_{1}, \ldots, g_{n}\right): \mathbb{R}^{n} \rightarrow \mathbb{R}^{n}$ is qmi if and only if it has the following property [14]: If $x, y \in \mathbb{R}^{n}$ and $k \in\{1, \ldots, n\}$ are such that $x \leq y$ and $x_{k}=y_{k}$ then $g_{k}(x) \leq g_{k}(y)$.

A function $f:[a, b] \times \mathbb{R}^{n} \rightarrow \mathbb{R}^{n}$ is called qmi in $x$ if $x \mapsto f(t, x)$ is qmi for each $t \in[a, b]$.

A connection between quasimonotonicity and differential inequalities is as follows [14].

Theorem 1 Let $f:[a, b] \times \mathbb{R}^{n} \rightarrow \mathbb{R}^{n}$ be qmi in $x$, and let $u, v:[a, b] \rightarrow \mathbb{R}^{n}$ be differentiable. Then

$$
u^{\prime}(t)-f(t, u(t)) \ll v^{\prime}(t)-f(t, v(t)) \quad(t \in[a, b]), \quad u(a) \ll v(a)
$$

imply $u(t) \ll v(t)(t \in[a, b])$.

Proof: Since $u, v$ are continuous with $u(a) \ll v(a)$ we have $u(t) \ll v(t)$ in a right neighbourhood of $a$. Assume that our assertion does not hold. Then there exists $t_{0} \in(a, b]$ such that $x:=v\left(t_{0}\right)-u\left(t_{0}\right) \in \partial K$ and $u(t) \ll v(t)(t \in$ $\left.\left[a, t_{0}\right)\right)$. Then $\langle x, \xi\rangle=0$ for some $0 \neq \xi \in K^{*}$ and therefore $\left\langle f\left(t_{0}, u\left(t_{0}\right)\right), \xi\right\rangle \leq$ $\left\langle f\left(t_{0}, v\left(t_{0}\right)\right), \xi\right\rangle$. The function $h:[a, b] \rightarrow \mathbb{R}$ defined by $h(t)=\langle v(t)-u(t), \xi\rangle$ is differentiable with $h^{\prime}\left(t_{0}\right) \leq 0$ since $h(t)>0\left(t \in\left[a, t_{0}\right)\right)$ and $h\left(t_{0}\right)=0$. On the other hand $\left.h^{\prime}\left(t_{0}\right)=\left\langle v^{\prime}\left(t_{0}\right)-u^{\prime}\left(t_{0}\right), \xi\right\rangle\right\rangle\left\langle f\left(t_{0}, v\left(t_{0}\right)\right)-f\left(t_{0}, u\left(t_{0}\right)\right), \xi\right\rangle \geq 0$.

Let us say that a function $f:[a, b] \times \mathbb{R}^{n} \rightarrow \mathbb{R}^{n}$ is locally Lipschitz continuous in $x$ if to each $r>0$ there is a constant $L_{r}$ such that

$$
\|f(t, x)-f(t, y)\| \leq L_{r}\|x-y\| \quad(t \in[a, b],\|x\|,\|y\| \leq r) .
$$

We will now give an elementary proof of a $\leq$ version of Theorem 1, see [14].

Theorem 2 Let $f:[a, b] \times \mathbb{R}^{n} \rightarrow \mathbb{R}^{n}$ be qmi and locally Lipschitz continuous in $x$, and let $u, v:[a, b] \rightarrow \mathbb{R}^{n}$ be differentiable. Then

$$
u^{\prime}(t)-f(t, u(t)) \leq v^{\prime}(t)-f(t, v(t)) \quad(t \in[a, b]), \quad u(a) \leq v(a)
$$

imply $u(t) \leq v(t)(t \in[a, b])$. 
Proof: Let $r>0$ such that $\|u(t)\|,\|v(t)\| \leq r-1(t \in[a, b])$. Since $f$ is locally Lipschitz continuous and according to the relations between norm and order there is a constant $L=L_{r}>0$ such that

$$
f(t, x)-f(t, y) \leq L\|x-y\| p \quad(t \in[a, b],\|x\|,\|y\| \leq r) .
$$

For $\varepsilon>0$ we consider the functions $h_{\varepsilon}:[a, b] \rightarrow \mathbb{R}$

$$
h_{\varepsilon}(t)=\varepsilon \exp (L(t-a))+\frac{\varepsilon}{L}(\exp (L(t-a))-1),
$$

which has the properties

$$
h_{\varepsilon}^{\prime}(t)=L h_{\varepsilon}(t)+\varepsilon, \quad h_{\varepsilon}(t) \geq \varepsilon
$$

for $t \in[a, b]$, and $h_{\varepsilon}(t) \rightarrow 0$ as $\varepsilon \rightarrow 0+$ uniformly on $[a, b]$. We set

$$
u_{\varepsilon}(t)=u(t)-h_{\varepsilon}(t) p, \quad v_{\varepsilon}(t)=v(t)+h_{\varepsilon}(t) p .
$$

We have $u_{\varepsilon}(a) \ll v_{\varepsilon}(a)$, and we choose $\varepsilon>0$ such that $h_{\varepsilon} \leq 1$ on $[a, b]$. Then $\left\|u_{\varepsilon}(t)\right\|,\left\|v_{\varepsilon}(t)\right\| \leq r(t \in[a, b])$ and therefore

$$
\begin{gathered}
u_{\varepsilon}^{\prime}(t)-f\left(t, u_{\varepsilon}(t)\right)=u^{\prime}(t)-h_{\varepsilon}^{\prime}(t) p-f(t, u(t))+f(t, u(t))-f\left(t, u_{\varepsilon}(t)\right) \\
\leq v^{\prime}(t)-f(t, v(t))-h_{\varepsilon}^{\prime}(t) p+L\left\|h_{\varepsilon}(t) p\right\| p \\
=v_{\varepsilon}^{\prime}(t)-f\left(t, v_{\varepsilon}(t)\right)-2 h_{\varepsilon}^{\prime}(t) p+f\left(t, v_{\varepsilon}(t)\right)-f(t, v(t))+L h_{\varepsilon}(t) p \\
\leq v_{\varepsilon}^{\prime}(t)-f\left(t, v_{\varepsilon}(t)\right)-2 h_{\varepsilon}^{\prime}(t) p+2 L h_{\varepsilon}(t) p \\
=v_{\varepsilon}^{\prime}(t)-f\left(t, v_{\varepsilon}(t)\right)-2 \varepsilon p \ll v_{\varepsilon}^{\prime}(t)-f\left(t, v_{\varepsilon}(t)\right)(t \in[a, b]) .
\end{gathered}
$$

By Theorem 1 we have $u_{\varepsilon}(t) \ll v_{\varepsilon}(t)(t \in[a, b])$. Hence $\varepsilon \rightarrow 0+$ leads to $u(t) \leq v(t)(t \in[a, b])$.

An immediate consequence of Theorem 1 is for example the monotone dependence of the solution of an initial value problem with qmi right hand side on the initial value. If $f:[a, b] \times \mathbb{R}^{n} \rightarrow \mathbb{R}^{n}$ is continuous and locally Lipschitz continuous in $x$ then the problems

$$
u^{\prime}(t)=f(t, u(t)), u(a)=u_{0}, \quad v^{\prime}(t)=f(t, v(t)), v(a)=v_{0}
$$

are uniquely locally solvable to the right. If $f$ is qmi in $x$ and $u_{0} \leq v_{0}$ then Theorem 2 gives $u(t) \leq v(t)$ as long as both solutions exist.

For differentiable functions the question wether $g$ is qmi or not can be reduced to the linear case. 
Theorem 3 Let $g: \mathbb{R}^{n} \rightarrow \mathbb{R}^{n}$ be differentiable on $\mathbb{R}^{n}$. Then $g$ is qmi if and only if $x \mapsto g^{\prime}(z) x$ is qmi for each $z \in \mathbb{R}^{n}$.

Proof: 1. Let $g$ be $q$ mi and fix $z \in \mathbb{R}^{n}$. Let $x \leq y$ and $\xi \in K^{*}$ such that $\langle x, \xi\rangle=\langle y, \xi\rangle$. Then for $t \in[0,1]$ we have $z \leq z+t(y-x)$ and $\langle z, \xi\rangle=\langle z+t(y-x), \xi\rangle$ and therefore $\langle g(z), \xi\rangle \leq\langle g(z+t(y-x)), \xi\rangle$. Now,

$$
\left\langle g^{\prime}(z)(y-x), \xi\right\rangle=\lim _{t \rightarrow 0+} \frac{\langle g(z+t(y-x))-g(z), \xi\rangle}{t} \geq 0
$$

that is $\left\langle g^{\prime}(z) x, \xi\right\rangle \leq\left\langle g^{\prime}(z) y, \xi\right\rangle$.

2. Let $x \mapsto g^{\prime}(z) x$ be qmi for each $z \in \mathbb{R}^{n}$. Again let $x \leq y$ and $\xi \in K^{*}$ such that $\langle x, \xi\rangle=\langle y, \xi\rangle$. By the Mean Value Theorem there exisis $t_{0} \in[0,1]$ such that

$$
\langle g(y)-g(x), \xi\rangle=\left\langle g^{\prime}\left(x+t_{0}(y-x)\right)(y-x), \xi\right\rangle \geq 0 .
$$

Hence $\langle g(x), \xi\rangle \leq\langle g(y), \xi\rangle$.

Thus, it is interesting to know which linear functions are qmi.

4. The linear case. Let us call a matrix $A \in \mathbb{R}^{n \times n}$ qmi if $x \mapsto A x$ is qmi. For $\mathbb{R}^{n}$ ordered by $K_{\text {nat }}$ it is easy to recognize qmi matrices by the signs of their entries. In this case $A=\left(a_{i j}\right)$ is qmi if and only if $a_{i j} \geq 0$ if $i \neq j$. For $K_{\text {ice }}$ it is more difficult to characterize the qmi matrices, but a characterization is known [12]:

Let $\mathbb{R}^{n}$ be ordered by $K_{\text {ice }}$ and let $Q \in \mathbb{R}^{n \times n}$ denote the diagonal matrix with first $n-1$ entries 1 and last entry -1 . Then $A$ is qmi if and only if $Q A+A^{\top} Q+\lambda Q$ is negative semidefinite for some $\lambda \in \mathbb{R}$. In case $n=3$ we get a class of matrices for which $A$ and $-A$ are qmi, see [12], namely if $A$ is of the form

$$
A=\left(\begin{array}{ccc}
\alpha & \beta & \gamma \\
-\beta & \alpha & \delta \\
\gamma & \delta & \alpha
\end{array}\right)
$$

For example each Jacobi matrix of

$$
g: \mathbb{R}^{3} \rightarrow \mathbb{R}^{3}, \quad g(x, y, z)=\left(2 x y,-x^{2}+y^{2}+z^{2}, 2 y z\right)
$$

is of this form and by Theorem 3 the functions $g$ and $-g$ are $q$ mi.

A difference to $K_{\text {nat }}$ is worth mentioning. If $A$ is qmi with respect to $K_{\text {nat }}$ then $A+\lambda I$ is monotone increasing if $\lambda$ is big enough. This cannot be said for $K_{\text {ice }}$ [8]: For example

$$
A=\left(\begin{array}{lll}
\lambda & 0 & 1 \\
0 & \lambda & 0 \\
1 & 0 & \lambda
\end{array}\right)
$$


is qmi but never monotone increasing, no matter how big $\lambda$ is chosen.

Let us return to the general case. If $A \in \mathbb{R}^{n \times n}$ is qmi, then $x \mapsto \exp (t A) x$ is monotone increasing for each $t \geq 0$. This follows by Theorem 2 since for $x_{0} \geq 0, u(t)=0$ and $v(t)=\exp (t A) x_{0}$

$$
u^{\prime}(t)-A u(t)=0=v^{\prime}(t)-A v(t)(t \geq 0), \quad u(a) \leq v(a)
$$

imply $\exp (t A) x_{0} \geq 0(t \geq 0)$. Likewise the converse is true. If $x \mapsto \exp (t A) x$ is monotone increasing for each $t \geq 0$, then $A$ is qmi. To see this consider $x_{0} \in K$ and $\xi \in K^{*}$ such that $\left\langle x_{0}, \xi\right\rangle=0$. Then

$$
\left\langle A x_{0}, \xi\right\rangle=\lim _{t \rightarrow 0+} \frac{\left\langle\exp (t A) x_{0}-x_{0}, \xi\right\rangle}{t} \geq 0
$$

Moreover if $A$ is qmi and $-A$ is qmi then $A^{2}$ is qmi. The reason is that $x_{0} \in K, \xi \in K^{*}$ and $\left\langle x_{0}, \xi\right\rangle=0$ imply $\left\langle A x_{0}, \xi\right\rangle=0$ and then

$$
\left\langle A^{2} x_{0}, \xi\right\rangle=\lim _{t \rightarrow 0+} 2 \frac{\left\langle\exp (t A) x_{0}-x_{0}-t A x_{0}, \xi\right\rangle}{t^{2}} \geq 0
$$

These considerations lead to a nice example of a qmi linear mapping in $\mathbb{R}^{2 n+1}$ odered by $K_{\text {pol }}$. We identify $\mathbb{R}^{2 n+1}$ and $P_{2 n}$, the space of all real polynomials with degree $\leq 2 n$. We consider $D: P_{2 n} \rightarrow P_{2 n}$ defined by $D q=q^{\prime}\left(q \in P_{2 n}\right)$. According to Taylor's formula

$$
(\exp (t D) q)(s)=q(t+s) \quad(t \in \mathbb{R})
$$

Hence $D$ and $-D$ are $q \mathrm{mi}$ and therefore the second derivative $D^{2}$ is qmi. If for example $q \geq 0$, the function $w:[0, \infty) \times \mathbb{R} \rightarrow \mathbb{R}, w(t, s)=\left(\exp \left(t D^{2}\right) q\right)(s)$ solves the Cauchy problem

$$
w_{t}(t, s)=w_{s s}(t, s), \quad w(0, s)=q(s)
$$

and is nonnegative since $D^{2}$ is qmi. Thinking of the integral representation for solutions of the Cauchy problem for the heat equation, $w(t, s) \geq 0$ is obvious. But we did not need this representation.

5. Zeros, fixed points and intermediate values. We consider a continuously differentiable and qmi function $g: \mathbb{R}^{n} \rightarrow \mathbb{R}^{n}$. For a scalar initial value problem, the solution of $y^{\prime}(t)=g(y(t)), y(0)=y_{0}$ is increasing if $g\left(y_{0}\right) \geq 0$ and decreasing if $g\left(y_{0}\right) \leq 0$. The same result holds to the right for $n>1$, see [11], p.34. 
Theorem 4 For $T>0$ let $y:[0, T] \rightarrow \mathbb{R}^{n}$ solve $y^{\prime}(t)=g(y(t))$. Then $y$ is increasing if $g(y(0)) \geq 0$ and decreasing if $g(y(0)) \leq 0$.

Proof: Consider the case $g(y(0)) \geq 0$. We have $y^{\prime \prime}(t)=g^{\prime}(y(t)) y^{\prime}(t)(t \in$ $[0, T])$ and $y^{\prime}(0)=g(y(0))$. The function $f:[0, T] \times \mathbb{R}^{n} \rightarrow \mathbb{R}^{n}, f(t, x)=$ $g^{\prime}(y(t)) x$ is qmi in $x$ according to Theorem 3 . For $u, v:[0, T] \rightarrow \mathbb{R}^{n}$ defined by $u(t)=0$ and $v(t)=y^{\prime}(t)$ we have

$$
u^{\prime}(t)-f(t, u(t))=0=v^{\prime}(t)-f(t, v(t))(t \in[0, T]), \quad u(0) \leq v(0) .
$$

By Theorem 2, $u(t) \leq v(t)$, that is $0 \leq y^{\prime}(t)(t \in[0, T])$. Now, let $0 \leq$ $t_{1} \leq t_{2} \leq T$. If $y\left(t_{1}\right) \leq y\left(t_{2}\right)$ does not hold, there is some $\xi \in K^{*}$ such that $\left\langle y\left(t_{1}\right)-y\left(t_{2}\right), \xi\right\rangle<0$. But $\langle y(t), \xi\rangle$ is increasing, a contradiction.

Theorem 4 is a key to results on zeros and fixed points of qmi functions.

Theorem 5 Let $u_{0}, v_{0} \in \mathbb{R}^{n}$ be such that

$$
u_{0} \leq v_{0}, \quad g\left(u_{0}\right) \geq 0, \quad g\left(v_{0}\right) \leq 0
$$

Then $g$ has a zero in the order interval $\left[u_{0}, v_{0}\right]$.

Proof: Let $u, v$ be the solutions of $y^{\prime}(t)=g(y(t))$ with inital value $y(0)=u_{0}$ and $y(0)=v_{0}$, and with the maximal interval of existence $\left[0, \omega_{u}\right)$ and $\left[0, \omega_{v}\right)$, respectively. By Theorem $2, u(t) \leq v(t)$ as long as both solutions exist. By Theorem $4, u$ is increasing and $v$ is decreasing. In particular $u$ and $v$ do not leave $\left[u_{0}, v_{0}\right]$ and therefore $\omega_{u}=\omega_{v}=\infty$. Since $u$ is increasing and $u(t) \leq v_{0}$ $(t \geq 0)$ the limit $x_{0}:=\lim _{t \rightarrow \infty} u(t)$ exists; it is a zero of $g$.

Theorem 5 can be transformed to a fixed point theorem or an intermediate value theorem:

Theorem 6 Let $u_{0}, v_{0} \in \mathbb{R}^{n}$ be such that $u_{0} \leq v_{0}, g\left(u_{0}\right) \geq u_{0}, g\left(v_{0}\right) \leq v_{0}$. Then $g$ has a fixed point in the order interval $\left[u_{0}, v_{0}\right]$.

Proof: Apply Theorem 5 to $x \mapsto g(x)-x$.

Theorem 7 Let $u_{0}, v_{0} \in \mathbb{R}^{n}$ be such that $u_{0} \leq v_{0}, g\left(v_{0}\right) \leq g\left(u_{0}\right)$. Then $\left[g\left(v_{0}\right), g\left(u_{0}\right)\right] \subseteq g\left(\left[u_{0}, v_{0}\right]\right)$. 
Proof: Choose $y_{0} \in\left[g\left(v_{0}\right), g\left(u_{0}\right)\right]$ and apply Theorem 5 to $x \mapsto g(x)-y_{0}$.

Finally let us discuss an example to Theorem 6 . Let $\mathbb{R}^{2}$ be ordered by $K_{\text {nat }}$ and consider $g: \mathbb{R}^{2} \rightarrow \mathbb{R}^{2}$ defined by

$$
g(x, y)=\left(-2 \exp \left(-y^{3}\right) \cos ^{2}(x),-\exp \left(-x^{5}\right) \cos ^{4}(y)\right)
$$

This function is qmi since $y \mapsto-2 \exp \left(-y^{3}\right) \cos ^{2}(x)$ is increasing for each $x$ and $x \mapsto-\exp \left(-x^{5}\right) \cos ^{4}(y)$ is increasing for each $y$.

For $u_{0}=(-\pi / 2,-\pi / 2) \leq(0,0)=v_{0}$ we have

$$
g\left(u_{0}\right)=(0,0) \geq(-\pi / 2,-\pi / 2)=u_{0}, \quad g\left(v_{0}\right)=(-2,-1) \leq(0,0)=v_{0} .
$$

By Theorem 6 there is a fixed point of $g$ in $\left[u_{0}, v_{0}\right]$. Since $(-2,-1)$ is not in $\left[u_{0}, v_{0}\right]$, Brouwer's Fixed Point Theorem cannot be applied.

6. Further reading. Comprehensive texts focusing differential inequalities are for example [7], [13] and [17]. Generalizations of Theorem 2 can also be found in [10] and [15]. For a survey on monotonicity and quasimonotonicity methods for ODEs in infinite dimensional Banach spaces see [16]. The monograph [11] provides a dynamical system view on ODEs with qmi right hand side with many applications, for example to population dynamics. For the linear case in a Banach algebra setting see [4], and for positive semigroups we refer to [1]. Some papers on fixed points and related questions are [3], [5], $[6]$, and $[9]$.

\section{References}

[1] Arendt, W., Grabosch, A., Greiner, G., Groh, U., Lotz, H.P., Moustakas, U., Nagel, R., Neubrander, F., Schlotterbeck, U.: One-parameter semigroups of positive operators. Lect. Notes Math. 1184 (1986).

[2] Deimling, K.: Nonlinear Functional Analysis. Springer-Verlag, Berlin Heidelberg - New York - Tokyo, 1985.

[3] Herzog, G.: A fixed point theorem for quasimonotone increasing mappings. Acta Sci. Math. 64 (1998), 293-297.

[4] Herzog, G., Lemmert, R.: On quasipositive elements in ordered Banach algebras. Stud. Math. 129 (1998), 59-65. 
[5] Herzog, G., Lemmert, R.: Intermediate value theorems for quasimonotone increasing mappings. Numerical Funct. Anal. Opt. 20 (1999), 901908.

[6] Hu, S.: Fixed points for dicontinuous maps in $\mathbb{R}^{n}$. Proc. Amer. Math. Soc. 104 (1988), 1111-1114.

[7] Lakshmikantham, V., Leela, S.: Differential and Integral Inequalities I. Academic Press, New York - London, 1969.

[8] Lemmert, R., Volkmann, P.: On the positivity of semigroups of operators. Comment. Math. Univ. Carolinae 39 (1998), 483-489.

[9] Redheffer, I., Volkmann, P.: Ein Fixpunktsatz für quasimonoton wachsende Funktionen. Arch. Math. 70 (1998), 307-312.

[10] Redheffer, R.M.: Gewöhnliche Differentialungleichungen mit quasimonotonen Funktionen in normierten linearen Räumen. Arch. Rational Mech. Analysis 52 (1973), 121-133.

[11] Smith, H.L.: Monotone Dynamical Systems. AMS, Providence, Rhode Island, 1995.

[12] Stern, R.J., Wolkowicz, H.: Exponential nonnegativity on the ice cream cone. SIAM J. Matrix Anal. Appl. 12 (1991), 160-165.

[13] Szarski, J.: Differential Inequalities. PWN, Warszawa, 1965.

[14] Volkmann, P.: Gewöhnliche Differentialungleichungen mit quasimonoton wachsenden Funktionen in topologischen Vektorräumen. Math. Z. 127 (1972), 157-164.

[15] Volkmann, P.: Gewöhnliche Differentialungleichungen mit quasimonoton wachsenden Funktionen in Banachräumen. Lect. Notes Math. 415 (1974), 439-443.

[16] Volkmann, P.: Cinq cours sur les équations différentielles dans les espaces de Banach. Topological Methods in Differential Equations and Inclusions, Kluwer Acad. Publ., Dodrecht - Boston - London, 1995.

[17] Walter, W.: Differential and Integral Inequalities. Springer-Verlag, Berlin - Heidelberg - New York, 1970. 
Gerd Herzog, Roland Lemmert

Mathematisches Institut I

Universität Karlsruhe

D-76128 Karlsruhe

Germany

e-mail: Gerd.Herzog@math.uni-karlsruhe.de

e-mail: Roland.Lemmert@math.uni-karlsruhe.de 